\title{
Electrically induced Bragg Reflectors in InP/InGaAsP waveguides as ultrafast optoelectronic modulators
}

\section{Martina De Laurentis}

\author{
Andrea Irace \\ a.irace@unina.it
}

\section{Giovanni Breglio}

\author{
Department of Electronics and Telecommunication Engineering, \\ University of Naples "Federico II" Via Claudio, 21, I-50125 Naples, Italy \\ Department of Electronics and Telecommunication Engineering, \\ University of Naples "Federico II" Via Claudio, 21, I-50125 Naples, Italy \\ Department of Electronics and Telecommunication Engineering, \\ University of Naples "Federico II" Via Claudio, 21, I-50125 Naples, Italy
}

In this paper, with the help of numerical simulations, we show how it is possible to create a Distributed Bragg Reflector by electrically inducing periodical refractive index variations in unperturbed III-V rib-waveguides to design different kind of devices. This approach allows to induce the optoelectronic devices by a suitable reconfiguration of the potential applied to the electrode matrix, i.e. to have a defined routing/switching matrix, or, for dynamic applications, realize intensity modulators, capable of reaching a theoretical $40 \mathrm{CHz}$ switching speed. [DOI: 10.2971/jeos.2008.08021]

Keywords: Bragg grating, optoelectronic modulator, electro-optic effects

\section{INTRODUCTION}

When a suitable electric field is applied to a semiconductor, different types of physical effects can induce a refractive index variation depending on field intensity and/or direction. The main are the non-linear and the quadratic electro-optic effect, related directly to electric field strength [1] and the carrier induced effects (Band-filling, Plasma effect and Bandgap Shrinkage effect, related to the change of the free carriers concentration due to inter-band, intra-band transitions and to the bands modification respectively) [2]-[5]. In III-V compounds and particularly in the InP based materials, these effects are all large enough to induce, for example, a Distributed Bragg Reflector [6] in an unperturbed rib-waveguide. The attractiveness of III-V direct-bandgap semiconductor materials in the optical communication application based on InP is the possibility to have a monolithic integration of the optical functionalities with electronic circuitry [7] and, in addition, the quaternary (III-V) alloy InGaAsP lattice, matched to InP, offers the possibility to tune the bandgap for different wavelength windows by adjusting its composition. Exploiting these features, we want to show how it is possible to induce a modulation of the light intensity in a III-V rib-waveguide by means of an induced Bragg Reflector (BR) to design different kind of devices by means of application of an external electric field. What is interesting is that the devices are induced, without the need of a particular geometry, in an unperturbed host ribwaveguide; in this way it is possible to design a general configuration of electrodes which, driven by a suitable potential distribution, allows for the creation of a particular light path, or to reach a desired intensity modulation or phase shift and so on to realize, as an example, a router or a wavelength multiplexer/demultiplexer.

\section{DEVICE DESCRIPTION}

The structure of the proposed device is shown in Figure 1. It is a vertical InP/InGaAsP p-i-n diode where the InGaAsP region, that is the one with the lower doping, defines the optical channel. The anode electrode has a peculiar comb-structure needed to induce the periodical refractive index modulation. Waveguide geometries, guiding layer thickness and height, have been chosen in order to sustain the single-mode condition and grating period $\Lambda=236 \mathrm{~nm}$ has been chosen in order to obtain a first order Bragg grating, that is according to the Bragg resonance condition

$$
\Lambda=\frac{\lambda_{0}}{2 n_{e f f}}
$$

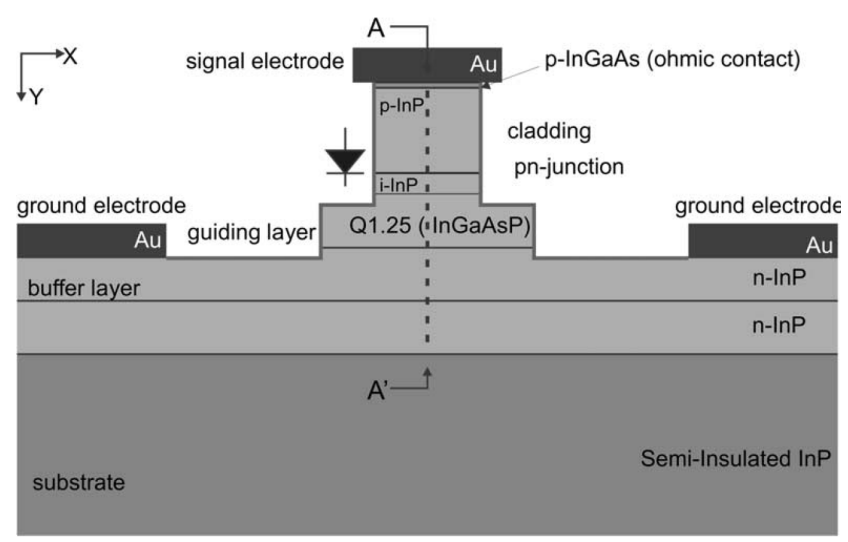



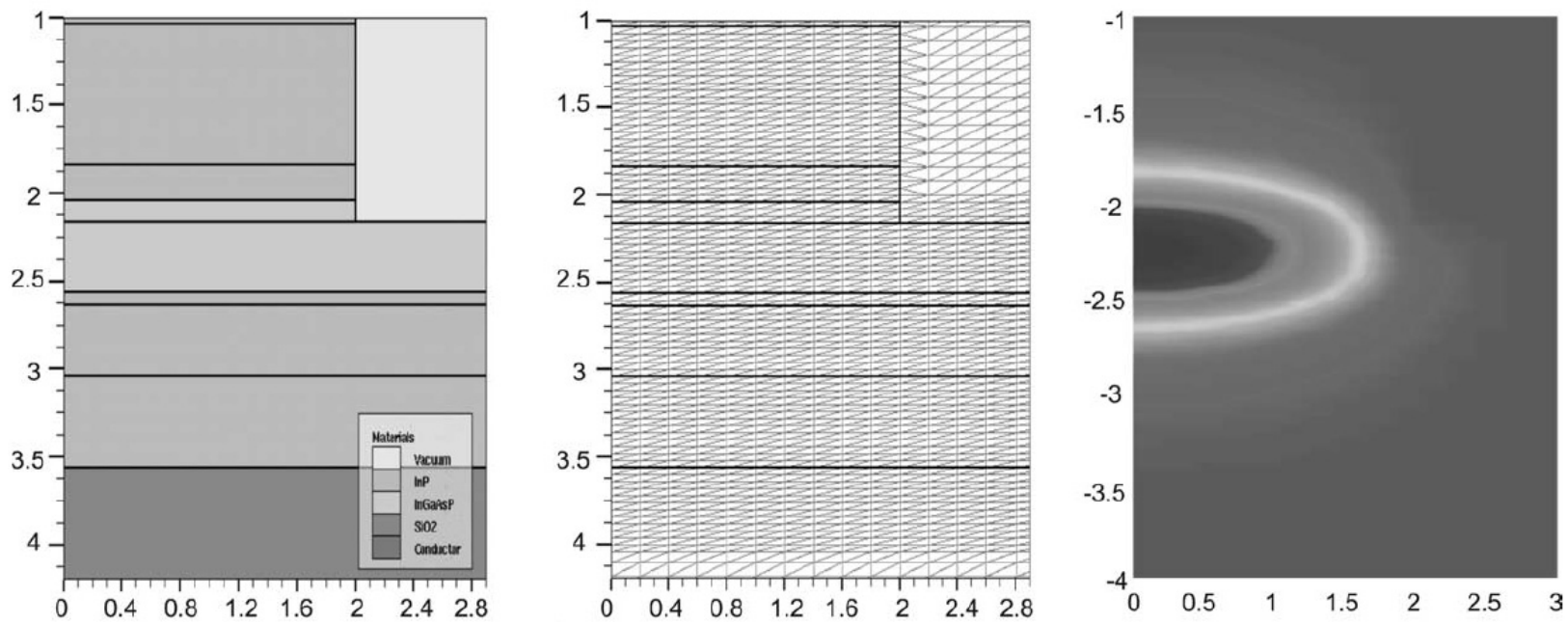

FIG. 2 From left to right: details of the section structure; corresponding Atlas grid and propagating fundamental TE mode as computed by FEM simulator.

where $\lambda_{0}$ is the wavelength in vacuum of the optical radiation traveling inside the device and $n_{e f f}$ is the effective refractive index of its first fundamental mode. The InP refractive index is $n_{\text {InP }}=3.167$ while for InGaAsP guiding layer we have $n_{\text {InGaAsP }}=3.39$ while optical absorption for both materials at $\lambda_{0}=1.55 \mu \mathrm{m}$ is negligible. The principle of operation of the device is straightforward: without biasing the diode, the Bragg Reflector is not induced and the light can travel in the modulator without being affected by any relevant loss; on the other hand, when a negative voltage is applied between anode and cathode, due to the periodic refraction index variation induced in the intrinsic region by the anode spatial periodicity, the Bragg mirror is formed along the waveguide and it affects light propagation in the optical channel. In the following, after an overview on the simulation strategy, we show numerical results regarding the performances of the Induced Bragg Reflector Amplitude Modulator (IBRAM). To design our electrically induced devices, we need to estimate the contributes of each of the electro-optic effects to the refractive index change as function of the anode applied voltage. The carriers induced effect can be evaluated by means of an electronic device simulator, together with the induced electric field inside the waveguide, from which we can directly obtain the contribution of the electro-optic effect, known the Pockels and Kerr coefficients and characteristic of the material (see [1]). Once done this, by means of an general purpose finite elements (FE) code the Helmholtz equation is solved and the effective refractive index is evaluated to predict the reflectance (transmittance) of the induced device. To this purpose we resort to and improve an in-house code [8], which combines the state-of-the-art electronic simulator Silvaco/ATLAS and a general purpose FE solver such as Comsol Multiphysics; this choice allows us to use the same simulation grid to evaluate all the quantities of interest, the effective index included. This MATLAB code takes as inputs the geometry of the section of the device, the mesh grid and computed carriers concentration together with the electric field evaluated on this grid by the device simulator and elaborates these data as outputs to Comsol Multiphysics to compute the effective refractive index of the rib guide fundamental TE and TM modes as function of the voltage applied on the electrodes. It thereafter predicts the
Bragg Grating reflectance, using the impedance matching matrix method [9].

\section{SIMULATION RESULTS}

In Figure 2 from left to right are reported the details of the structure shown in Figure 2, the corresponding FEM grid and the intensity distribution of the propagating fundamental TE mode evaluated by the FEM simulator on the same mesh grid. In Figure 3 the contributions to refractive index change of the various electro-optic effects vs the anode voltage are reported.

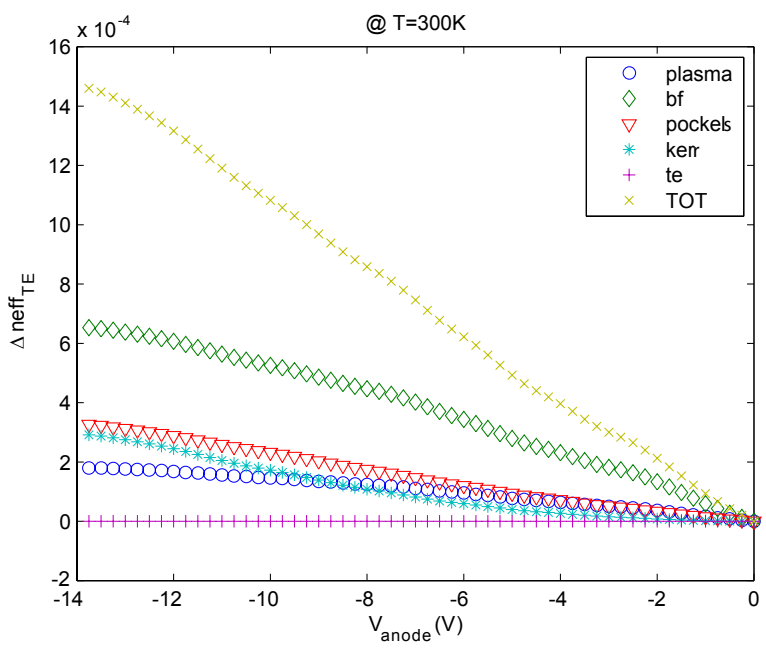

FIG. 3 Contribution to effective refractive index variation of the different e-o effects in the rib waveguide of Figure 2.

In this section we report an example of an electrically induced Bragg Modulator in a $2.5 \mathrm{~mm}$ long $\mathrm{InP} / \mathrm{InGaAsP}$ rib waveguide, with predicted switching speed higher than $40 \mathrm{GHz}$.

The device, in its cross section, refers to the structure described in the previous paragraph with a length optimized to have the shortest possible device with the smallest applied anode voltage. At this point we must note that there is a twofold approach in the choice of the maximum bias to be applied to the device. It is clear that higher voltages increase the elec- 


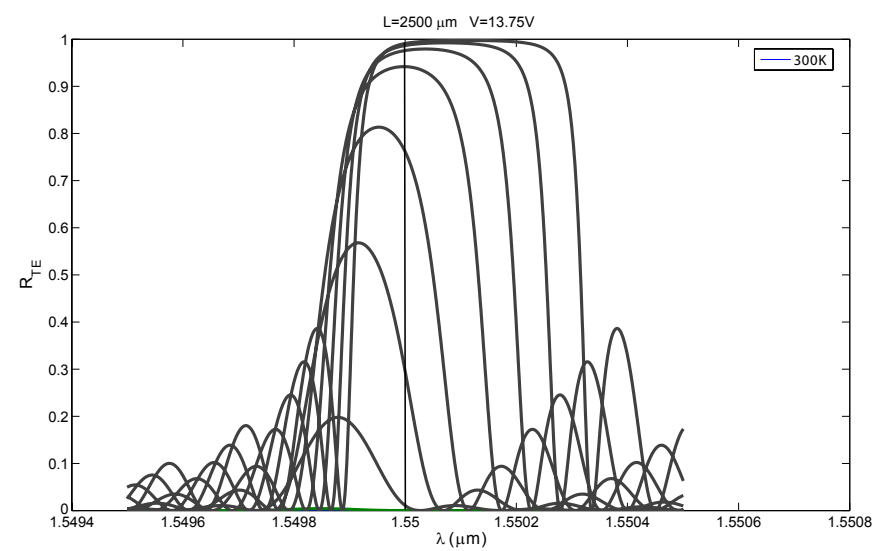

FIG. 4 Induced Bragg mirror Reflectivity spectrum changing the reverse bias applied between 0 and -10 V. At fixed wavelenght of $1.55 \mu \mathrm{m}$ it changes from 0 to about 1 .

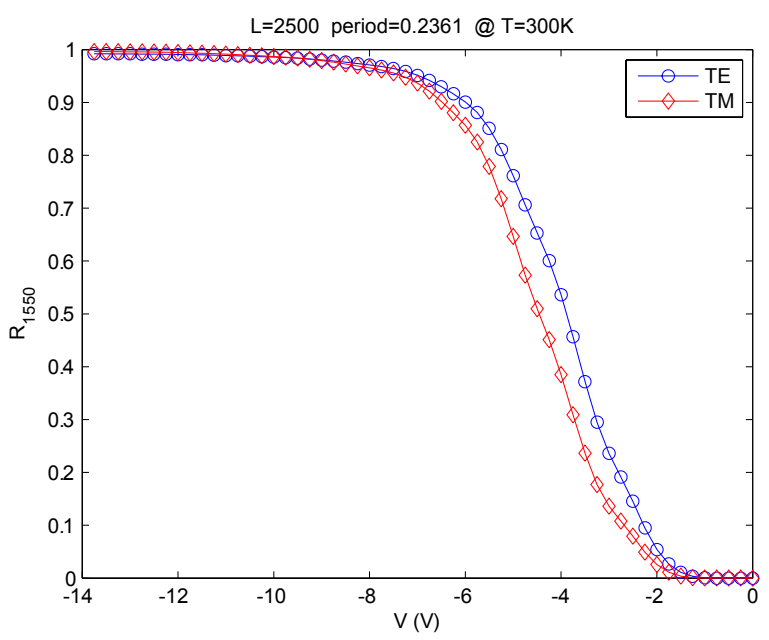

FIG. 5 Reflectivity at $1.550 \mu \mathrm{m}$ of the modulator against reverse bias applied for TE and TM polarization.

tric field and therefore the phase-shift per unit length. On the other hand as we increase the negative bias we get closer to avalanche breakdown of the junction and consequent injection of free carriers within the optical channel. The other choice is to make the device longer and reduce consequently the applied voltage; in this case we have to tolerate higher propagation losses. By trading off these two constraints we choose a length of $2.5 \mathrm{~mm}$ and a maximum bias of $-10 \mathrm{~V}$ applied on the anode. We emphasize that, due to the comb-like structure of the anode contact, a $2.5 \mathrm{~mm}$-long device presents the same parasitic capacitance of a $1.25 \mathrm{~mm}$-long full-covered electrode being therefore easier to drive by the electrical circuits. For a $2.5 \mathrm{~mm}$ long device, the induced Bragg Grating Reflectivity spectrum is shown in Figure 4: increasing the reverse voltage, the light at $1.55 \mu \mathrm{m}$ is totally reflected by the mirror and on-off modulation is obtained for applied voltages between 0 and $10 \mathrm{~V}$. In order to clarify the DC behavior of this modulator we report the optoelectronic transfer curve, that is the relationship between reflectivity and applied voltage, for both polarizations in Figure 5.

To verify the theoretical switching capability of this device, a negative square voltage pulse has been applied to the anode and reflectivity has been evaluated. The result of this simula-

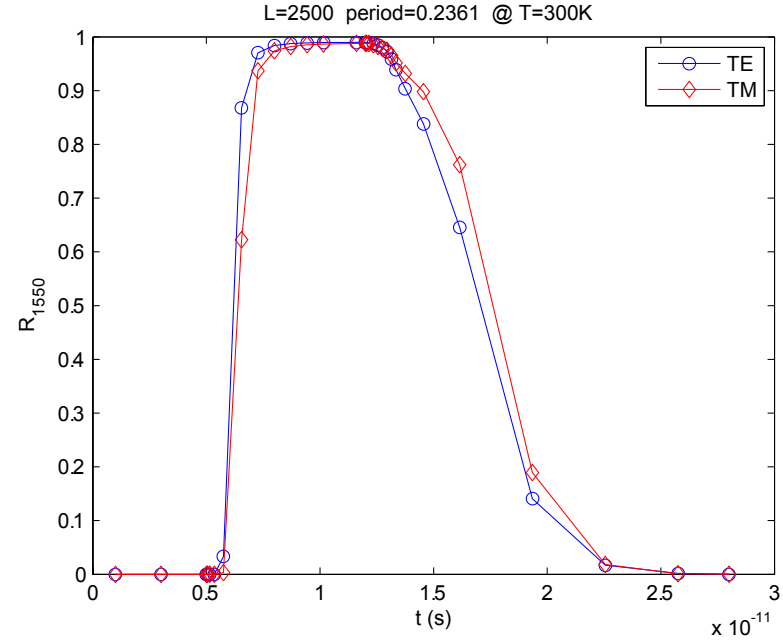

FIG. 6 Transient behavior of the proposed modulator when driven by a 8 ps long - $10 \mathrm{~V}$ square pulse. Rise times estimated of about 2 ps.

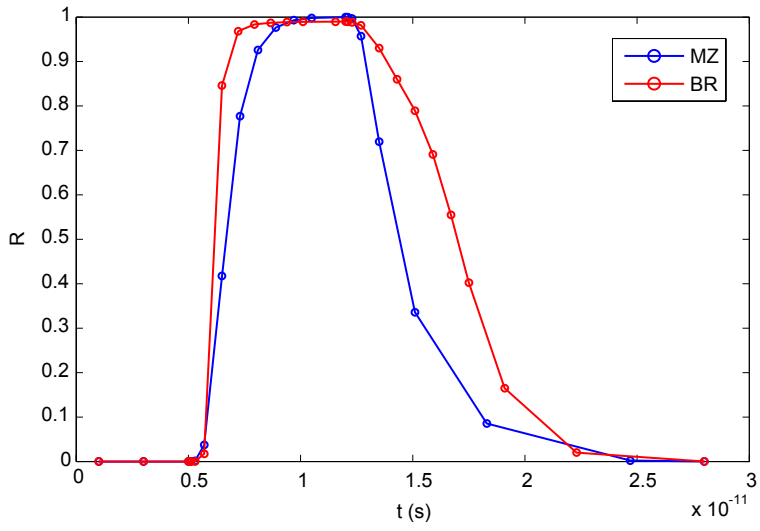

FIG. 7 Comparison of the transient response to an 8 ps long $-3.1 \mathrm{~V}$ pulse of a MachZehnder modulator and of the proposed Bragg modulator with the same section and active region length $(2.5 \mathrm{~mm})$.

tion is reported in Figure 6. We can observe the very fast rise and fall transient (which are of the order of 2 ps and 9 ps respectively) which predict the possible use of this device for ultra 40 GBit/s modulation rate. This switching speed is obviously a theoretical upper limit because of the driving amplifiers that have to be impedance matched to the modulator but this issue is not any different from driving of all kinds of optoelectronic modulators. Finally we compare the response of described Bragg modulator to the one of a Mach-Zehnder (MZ) configuration with the same device section cross-section and the same active region length $(2.5 \mathrm{~mm})$. In Figure 7 the transient response to the same pulse are shown. Though the voltage applied for a complete reflectivity on/off switch is lower $(3.1 \mathrm{~V})$, the transient response to the same pulse is slightly slower. We observe a risetime of about 4 ps against 2 ps of Bragg configuration while fall times are comparable. Besides, it must be noted that the same length correspond in the case of Bragg Modulator to half of the electrode capacitance which implies a faster response or an easier to design driver. This holds true also in push-pull configuration of the MZ modulator, even if in this case the length of the device is half respect the IBRAM. It is superfluous to note that the area on the chip of a push-pull $\mathrm{MZ}$ modulator and of a BR with the same ac- 
tive region is about the same, since the former is shorter, but double in width.

To further reduce the side-lobes of the Reflectivity Spectra of the grating, apodization [10] could be made, in order to prevent a modulator response increase at low bias, due to an undesirable shift of the spectra (for example by means of temperature change) or to reduce the device reflection band, if we want a more selective response, for example to have an filter instead of a modulator. In this kind of device obviously the apodization could not be a refractive index modulation as in optical fibers, but it must be realized as a modulation either of the grating period (as an example, an effective method could be the Randomly Sampled Apodization, RASA, [11]), or by means of the electrode periodicity duty-cycle. Both the cited methods can be easily realized in the fabrication process by means of a computer controlled Focused Ion Beam (FIB), which is an inspection tool usually present in the electronic devices technology [12].

\section{CONCLUSIONS}

We have shown how it is possible to design a $40 \mathrm{GHz}$ switching speed modulator by inducing a Bragg Reflector with a reverse biased vertical InP/InGaAsP $p$ - $i$ - $n$ diode. The predictions of grating reflectivity spectra and of the transient response have shown the capabilities of this device and its attractiveness as compared to other optical configurations such as Mach-Zehnder modulators.

\section{References}

[1] A. Yariv, Quantum Electronic - 3th edition (J Wiley a Sons 1987).
[2] S. Adachi, "Materials parameters of ingaasp and related binaries" J. Appl. Phys. 53, 8775 (1982).

[3] B. Bennet, "Carrier-induced change in refractive index of InP, CaAs and InGaAsP" IEEE J. Quantum. Elect. 26, 116 (1990).

[4] F. Fiedler, and A. Schlachetzk, "Optical parameters of inp-based waveguides" Solid State Electron. 30, 73 (1987).

[5] J.F. Vinchant, J.A. Cavailles, M. Erman, P. Jarry, and M. Renaud, "InP/GalnAsP guided-wave phase modulators based on carrierinduced effects: Theory and experiment" J. Lightwave Technol. 1, 63 (1992).

[6] A. Yariv, and M. Nakamura, "Periodic structures for integrated optics" IEEE J. Quantum. Elect. 13, 233 (1977).

[7] M. Smit, "InP photonic integrated circuits" in Proceedings of the 15th Annual Meeting of the IEEE Lasers and Electro-Optics Society (LEOS) 843 (2002).

[8] F. De Paola, V. d'Alessandro, A. Irace, J. den Besten, and M.Smit, "Novel optoelectronic simulation strategy of an ultrafast InP/InGaAsP modulator" 0pt. Commun. 256, 326 (2005).

[9] K. Winick, "Effective index method and coupled-mode theory for almost periodic waveguide gratings: a comparison" Appl. Opt. 31, 757 (1992).

[10] T. Erdogan, "Fiber grating spectra" J. Lightwave Technol. 15, 1277 (1997).

[11] M. Tormen, S. Ghidini, P. Crespi, D. Chacon, A. Nottola, S. Sardo, D. Crippa, M. Di Muri, G. Zuliani, and F. Giacometti, "Randomly sampled apodization in Bragg gratings" J. Lightwave Technol. 24, 1896 (2006).

[12] S. Reyntjens, and R. Puers, "Focused ion beam induced deposition: fabrication of three-dimensional microstructures and Young's modulus of the deposited material" J. Micromech. Microeng. 10, 181 (2000). 